\title{
Development of Inhalable Superparamagnetic Iron Oxide Nanoparticles (SPIONs) in Microparticulate System for Antituberculosis Drug Delivery
}

\author{
Margarida S. Miranda, Márcia T. Rodrigues, Rui M. A. Domingues, Rui R. Costa, \\ Elvira Paz, Carlos Rodríguez-Abreu, Paulo Freitas, Bernardo G. Almeida, \\ Maria Alice Carvalho, Carine Gonçalves, Catarina M. Ferreira, Egídio Torrado, \\ Rui L. Reis, Jorge Pedrosa, and Manuela E. Gomes*
}

Tuberculosis (TB) is an infectious disease which affects millions of people worldwide. Inhalable polymeric dry powders are promising alternatives as anti-TB drug carriers to the alveoli milieu and infected macrophages, with potential to significantly improve the therapeutics efficiency. Here, the development of a magnetically responsive microparticulate system for pulmonary delivery of an anti-TB drug candidate (P3) is reported. Microparticles (MPs) are developed based on a cast method using calcium carbonate sacrificial templates and incorporate superparamagnetic iron oxide nanoparticles to concentrate MPs in alveoli and enable drug on demand release upon actuation of an external alternate magnetic field (AMF). The MPs are shown to be suitable for P3 delivery to the lower airways and for alveolar macrophage phagocytosis. The developed MPs reveal unique and promising features to be used as an inhalable dry powder allowing the AMF control over dosage and frequency of drug delivery anticipating improved TB treatments.

\section{Introduction}

Tuberculosis (TB) is an infection disease affecting about one third of the world's population. ${ }^{[1]}$ The pulmonary route is the principal route of infection that initiates when Mycobacterium tuberculosis (Mtb) bacilli are inhaled and phagocytized by alveolar macrophages. However, some bacilli are able to survive within macrophage phagosomes leading to granuloma tissue formation that hampers an efficient treatment.. ${ }^{[2]}$ The prolonged treatment regimens and the toxic side effects resultant from oral/injectable administration of multiple drugs and dosages lead to reduced patient compliance that further increases the chances of development of multidrug-resistant strains. ${ }^{[3]}$ Thus, there is an urgent demand
Dr. M. S. Miranda, Dr. M. T. Rodrigues, Dr. R. M. A. Domingues,

Dr. R. R. Costa, Prof. R. L. Reis, Prof. M. E. Gomes

3B's Research Group

I3Bs-Research Institute on Biomaterials, Biodegradables

and Biomimetics

University of Minho

Headquarters of the European Institute of Excellence on Tissue

Engineering and Regenerative Medicine

AvePark, Parque de Ciência e Tecnologia

Zona Industrial da Gandra, 4805-017 Barco, Guimarães, Portugal

E-mail: megomes@i3bs.uminho.pt

Dr. M. S. Miranda, Dr. M. T. Rodrigues, Dr. R. M. A. Domingues,

Dr. R. R. Costa, Dr. E. Paz, Dr. C. Rodríguez-Abreu, Dr. P. Freitas,

Prof. B. G. Almeida, Prof. M. A. Carvalho, Dr. C. Gonçalves, C. M. Ferreira,

Dr. E. Torrado, Prof. R. L. Reis, Prof. J. Pedrosa, Prof. M. E. Gomes

ICVS/3B's - PT Government Associate Laboratory

Braga/Guimarães, Portugal

Dr. M. S. Miranda, Dr. M. T. Rodrigues, Dr. R. M. A. Domingues,

Dr. R. R. Costa, Prof. R. L. Reis, Prof. M. E. Gomes

The Discoveries Centre for Regenerative and Precision Medicine Headquarters at University of Minho

Avepark, 4805-017 Barco, Guimarães, Portugal

The ORCID identification number(s) for the author(s) of this article can be found under https://doi.org/10.1002/adhm.201800124.

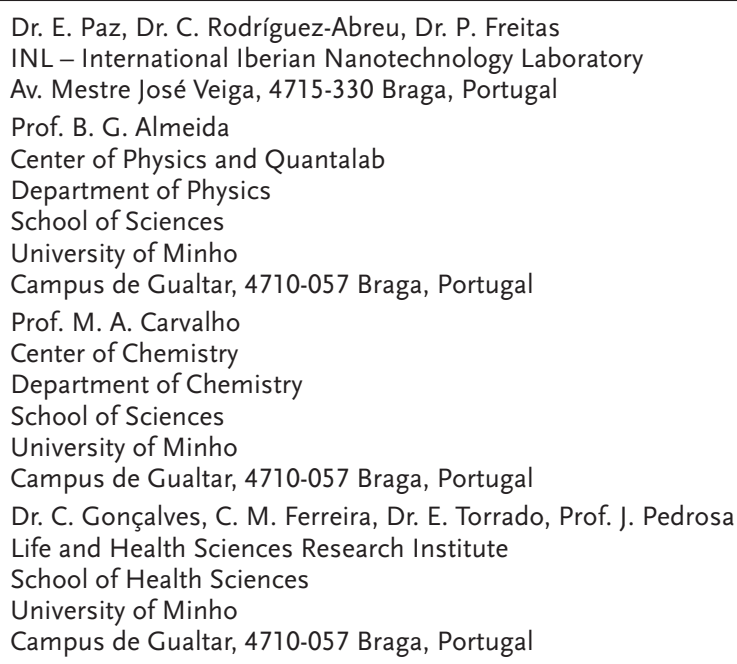


for new anti-TB drugs and improved carriers systems for their effective delivery into the lower airways and alveoli.

The administration of inhalable drugs offers advantages in comparison to conventional routes which include targeting drugs directly to the infected area increasing local drug concentration and reducing side effects. ${ }^{[4]}$ Inhalable carrier systems in opposition to pure drug formulations allow protection of drugs avoiding their early degradation and fast clearance. Moreover, drug carriers allow to control and sustain the release of drugs over long periods of time and improve drug uptake by alveolar macrophages. ${ }^{[5]}$

Dry powder formulations are currently being considered among the most promising delivery systems for pulmonary administration. ${ }^{[6]}$ As a result of their dehydrated form, dry powder formulations demonstrate higher physicochemical stability even under unrefrigerated conditions, thus extending their long-term storage outside of the cold chain. Furthermore, and unlike other inhaler devices, dry powder inhalers are propellant free, portable, easy to operate and cost-effective. However, inhalable formulations must encompass particular physicochemical properties which justify a considerable particulate engineering effort. For an effective deposition in the pulmonary alveoli, the optimal aerodynamic diameter of the particles should range from 1 to $5 \mu \mathrm{m} .{ }^{[7]}$ Moreover, when the physical diameter meets the phagocytic size range capacity of alveolar macrophages $(1-6 \mu \mathrm{m})$, it is possible to guide the particles into infected macrophages, which is advantageous for TB therapies as this approach leads to an intracellular influx of drug with potential to neutralize Mtb bacilli. ${ }^{[8]}$

This work aims to design and develop inhalable multifunctional microparticulate systems with magnetic responsiveness for an effective pulmonary delivery of an anti-TB drug candidate (P3), into alveoli and alveolar macrophages, as a new approach toward improved TB treatment. Purine derivatives have been described as antimycobacterial agents and P3 is a 9-arylpurine derivative shown to have relevant anti-TB activity and low toxicity in both bone-marrow derived macrophages (BMDMs) and VERO mammalian cells. ${ }^{[9]}$ The microparticles (MPs) were fabricated with gelatin and bovine serum albumin (BSA) using a "cast" strategy by replicating the structure of spherical and porous calcium carbonate $\left(\mathrm{CaCO}_{3}\right)$ templates. ${ }^{[10]}$ Gelatin is a biocompatible, biodegradable, nontoxic and cost-effective natural polymer that has already been proposed as carrier for pulmonary delivery of anti-TB drugs. ${ }^{[1]}$ Due to P3 hydrophobic character, the MPs were filled with BSA, a natural delivery vehicle for hydrophobic drugs. ${ }^{[12]}$ To shape and stabilize the MPs before loading P3, MPs were crosslinked with genipin, a natural amine reactive cross-linker, reported to have lower cytotoxicity than traditional chemicals as glutaraldehyde. ${ }^{[10 c]}$

To provide magnetic responsiveness, superparamagnetic iron oxide nanoparticles (SPIONs) were incorporated into the MPs. The manipulation of magnetic elements such as SPIONs within inhalable systems were shown to enable guidance and concentration of drugs to a desired lung region, improving treatment's efficiency and minimizing side effects. ${ }^{[13]}$ Furthermore, SPIONs can be remotely activated under the influence of an external magnetic field allowing controlled drug delivery. ${ }^{[14]}$
Overall, the proposed system consisting of a new anti-TB drug in a finely tailored and magnetically responsive inhalable dry powder is expected to delivery P3 into the lower airways and to be phagocytized by macrophages. Moreover, the release profile of P3 can be triggered and modulated by the application of an external alternate magnetic field (AMF). Since the presence of SPIONs within MPs can be activated by magnetotherapy devices triggering drug release with the desired dosage and frequency, this approach is envisioned to contribute for improved efficiency of future TB treatments using pharmacological drugs.

\section{Results and Discussion}

\subsection{Characterization of Unloaded MPs}

The drug carrier shape and size are extremely important parameters in pulmonary drug delivery to ensure well-defined aerodynamic properties and also for the phagocytic process of MPs. Thus, unloaded MPs were freeze dried and their shape and size evaluated by scanning electron microscopy (SEM) (Figure 1A1,A2). SEM analysis showed that MPs display the spherical shape from the initial $\mathrm{CaCO}_{3}$ templates. Unlike previous reports on similar gelatin casted microgels produced without using BSA as filler, ${ }^{[10 c]}$ the developed MPs did not collapse after drying, keeping the desired spherical structure of the $\mathrm{CaCO}_{3}$ template. The histogram of the diameter frequency distribution of the dried MPs is shown in Figure 1B as along with their normal distribution function. Dry MPs present a diameter of $2.86 \pm 0.04 \mu \mathrm{m}(n=397)$ and a very narrow distribution of diameters. This highly monodispersed distribution results from the relatively uniform size of the porous $\mathrm{CaCO}_{3}$ template used to fabricate the MPs ${ }^{[10 c, d]}$ and represents an advantage over other production techniques that result in microparticle formulations with higher size distribution. ${ }^{[15]}$ The MPs size monodispersity is extremely important in drug delivery applications, and in particular in pulmonary drug delivery, not only to ensure uniform drug load and release profiles but also well-defined aerodynamic properties.

High-resolution SEM images also confirmed that the roughness from the porous structure of the $\mathrm{CaCO}_{3}$ (Figure 1C1,C2) template was well preserved in the gelatin microgels (Figure 1D1,D2) although the surface seems to be smother than that of $\mathrm{CaCO}_{3}$ templates. Besides the enabling the fine control over MPs dimension in the fabrication process, the use of a $\mathrm{CaCO}_{3}$ as porous template also aimed at increasing the MPs porosity and hence improving their dispersibility and aerodynamic performance through the increase of surface roughness and the number of contact points (cohesiveness) between MPs. ${ }^{[15 a]}$ Moreover, drug carriers with high bulk porosity generally have higher bulk surface area which leads to enhanced drug loading efficiency and favored release compared to compact structures obtained by other particle production techniques such as emulsification, which limit the absorption and subsequent release of encapsulated drugs. ${ }^{[10 c]}$

A number of complementary techniques were used to confirm the suitability of the fabrication process employed 

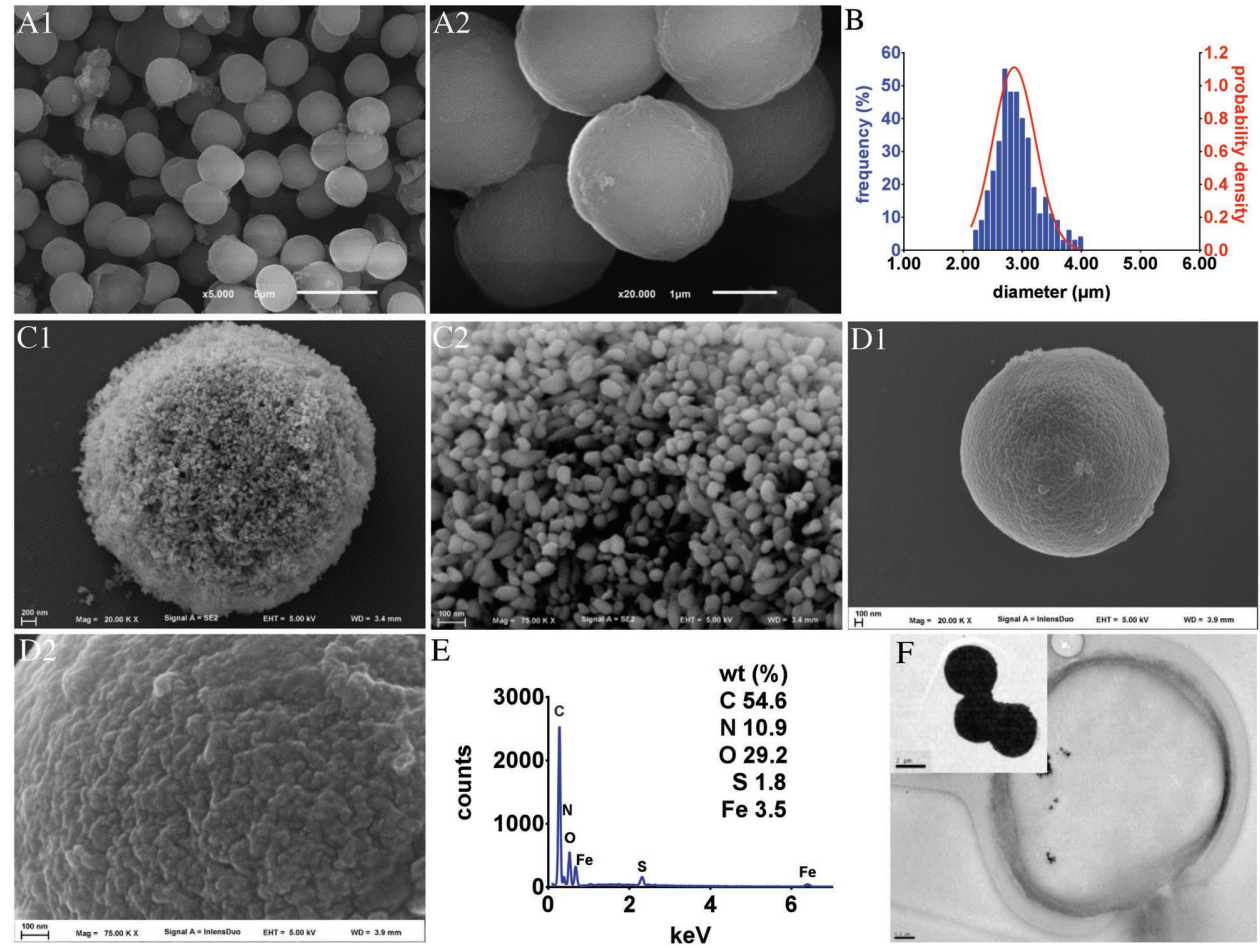

Figure 1. Shape, size, size distribution, and surface morphology of dried MPs. A) SEM images. B) Histogram of the distribution frequency of the diameters of dried MPs and normal distribution curve. C) High-resolution SEM images revealing the calcium carbonate porous surface topography. D) Highresolution SEM images revealing the MPs surface topography. E) EDS spectrum of MPs: elemental analysis: C $(54.6 \% \pm 1.3 \%)$, O (29.2\% $\pm 0.9 \%)$, $\mathrm{N}(10.9 \% \pm 1.8 \%), \mathrm{Fe}(3.5 \% \pm 0.3 \%), \mathrm{S}(1.8 \% \pm 0.1 \%)$. F) TEM image of the cross-section of an MP (scale bar represents $0.2 \mu \mathrm{m})$ where black dots represent magnetic nanoparticles; inset: image of MPs (scale bar represents $2 \mu \mathrm{m}$ ).

and the shape and size of the MPs. Complete $\mathrm{CaCO}_{3}$ template removal from the MPs was confirmed by energy dispersive spectroscopy (EDS) analysis as the calcium element is not detected on the particles (see Figure 1E). Additionally, EDS analysis also revealed the presence of iron element $(\mathrm{Fe})$ corresponding to the $\mathrm{Fe}_{3} \mathrm{O}_{4}$ core of SPIONs in the MPs. Transmission electron microscopy (TEM) images of the MPs are shown in Figure 1F and confirmed the spherical shape and the MPs diameter (inset of Figure 1F) assessed by SEM.
Furthermore, a cross-section analysis showed that SPIONs were both at the surface of the MPs, as assessed from EDS analysis, and incorporated within the MPs core (Figure 1F).

The developed MPs suspended in water also exhibited a spherical shape replicating the original form of the $\mathrm{CaCO}_{3}$ templates with a diameter of $4.19 \pm 0.05 \mu \mathrm{m} \quad(n=223)$ (Figure 2A1,A2). As expected, the diameter of the hydrated MPs was found to be higher than that of the dried MPs, $2.86 \pm 0.04 \mu \mathrm{m}$, due to the adsorption of water which causes
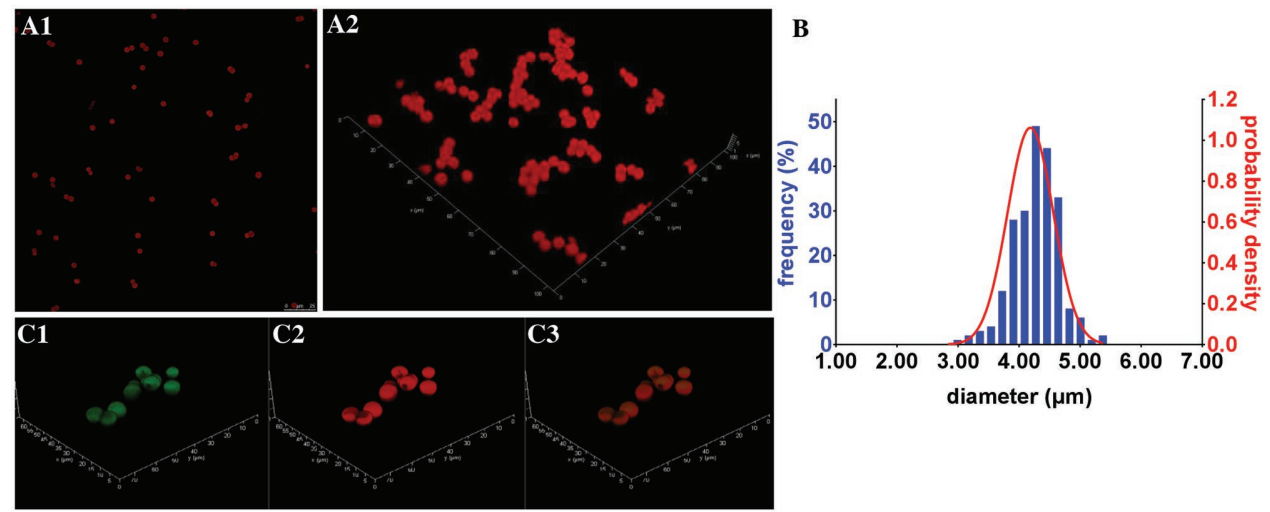

Figure 2. Assessment of shape, size, and size distribution of hydrated MPs by confocal microscopy. A) MPs produced with BSA are autofluorescent in the red channel $(560 \mathrm{~nm})$ : (A1) two dimension image, (A2) three dimension image. B) Histogram of the diameters frequency distribution of hydrated MPs and normal distribution curve. C) MPs produced with BSA-FITC showing fluorescence for the green channel (C1), red channel (C2), and overlay of green and red channels (C3). 
swelling of gelatin/BSA MPs. The frequency histogram of diameter distribution of the hydrated MPs is presented in Figure 2B along with the corresponding normal distribution function and as observed for the dried MPs, hydrated MPs also have a remarkably narrow size distribution.

To evaluate the carrier $\mathrm{pH}$ responsiveness, MPs were dispersed in phosphate buffered saline (PBS) 7.4 and 5.4 and their diameter analyzed by confocal microscopy. These $\mathrm{pH}$ values were chosen to mimic the $\mathrm{pH}$ under physiological conditions (7.4) and the $\mathrm{pH}$ in the macrophage phagosomes that are known to vary from 4.5 to $6.2 .^{[16]}$ The MPs were found to be $\mathrm{pH}$ sensitive exhibiting a decrease in diameter from $4.37 \pm 0.04 \mu \mathrm{m}$ $(n=342)$ at $\mathrm{pH} 7.4$ to $3.64 \pm 0.05 \mu \mathrm{m}(n=218)$ at $\mathrm{pH} 5.4$. A similar behavior has been previously described for gelatin microgels crosslinked with genipin. ${ }^{[10 c]}$ According to this study, most of the primary amine groups of the gelatin/BSA matrix are consumed in the crosslinking reaction with genipin and therefore the remaining ionizable groups are carboxylic acids (aspartic acid and glutamic acid) that have a lower degree of protonation at $\mathrm{pH} 7.4$ than at $\mathrm{pH}$ 5.4. The higher charge density of the MPs matrix at $\mathrm{pH} 7.4$ leads to an increased repulsion between protein chains within the microgel and consequently to higher swelling ratios.

In order to confirm the incorporation of BSA within the MPs, BSA-fluorescein isothiocyanate (FITC) instead of BSA was used in the MPs production process. The green fluorescence observed by confocal microscopy within the MPs (Figure 2C1) confirms the presence a relatively uniform distribution of BSA in the particles volume, which are autofluorescent in the red channel.

The shape and size of the MPs are particularly important features to meet the phagocytosis range of macrophages. Independently of their dry or hydrated form, MPs physical diameter is within the range of macrophages action, normally in the range of $1-6 \mu \mathrm{m} .{ }^{[8 \mathrm{~b}]}$

The SPIONs content in the MPs was quantitatively evaluated by thermal gravimetric analysis (TGA). Figure 3A represents a TGA experiment of dried MPs. Free SPIONs were used as experimental controls. In the MPs there is an initial mass loss due to the removal of strongly adsorbed water from the MPs. A second accentuated mass loss starting at about $200{ }^{\circ} \mathrm{C}$ corresponds to the combustion of organic

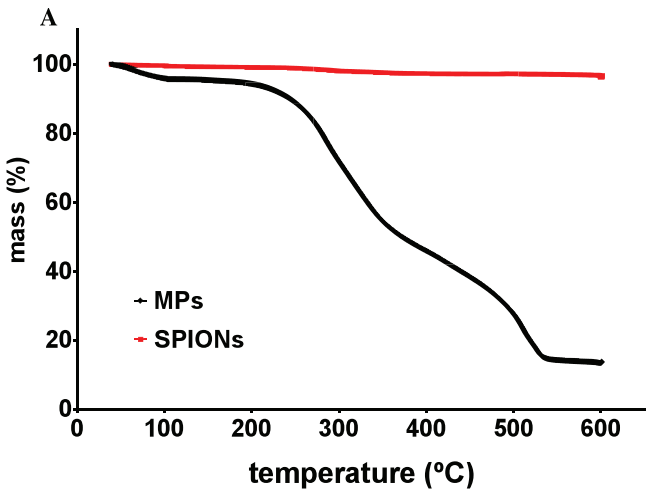

matter in the MPs. In free-SPIONs, only the initial water mass loss is detected. The mass percentage of SPIONs in the dry MPs was calculated to be $10.1 \% \pm 2.2 \% \mathrm{w} / \mathrm{w}$ (a correction was made for the mass loss of free SPIONs) and their water content was determined to be $3.0 \% \pm 0.8 \% \mathrm{w} / \mathrm{w}$ ( $n=6$, mean and two standard deviations from the mean of six experiments, for a level of confidence $95 \%$ ). The produced MPs have similar SPIONs content to other MPs formulations designed for pulmonary delivery. For example the ones reported by McBride et al. ${ }^{[15 a]}$ and Stocke et al. ${ }^{[15 b]}$ for targeted treatment of lung cancer.

The magnetic responsiveness of MPs was qualitatively confirmed by sorting the MPs from a water suspension using a permanent rare-earth magnet (inset of Figure 3B) and the magnetic behavior of dried MPs was assessed by SQUID (superconducting quantum interference device) analysis. The saturation magnetization of MPs at room temperature was determined to be $2.08 \mathrm{emu} \mathrm{g}^{-1}$, exhibiting zero coercive field and remanence magnetization at zero magnetic field (Figure 3B), which is consistent with a superparamagnetic behavior provided by the SPIONs incorporated within MPs. In superparamagnetic particles, a magnetic dipole moment is induced only when an external magnetic field is applied allowing the possibility of externally controlling the release of a loaded drug. As expected, the saturation magnetization value of the MPs is lower than that of free-SPIONs (46.01 emu g $\left.{ }^{-1}\right)$, reflecting their low magnetic material content $(10.1 \% \pm 2.2 \% \mathrm{w} / \mathrm{w})$.

\subsection{Characterization of Developed P3 Loaded MPs Systems}

\subsubsection{P3 Loading Assay}

A $48 \mathrm{~h}$ incubation period of MPs with a P3 drug solution was selected based on the preoptimization studies which indicated that this is the time required to attain the maximum loading (data not shown). The loading efficiency results are summarized in Table 1.

The loading efficiency of P3 into MPs was $82.7 \% \pm 0.9 \%$. This value is comparable to other dry powders produced by spray-drying and it is significantly higher than other

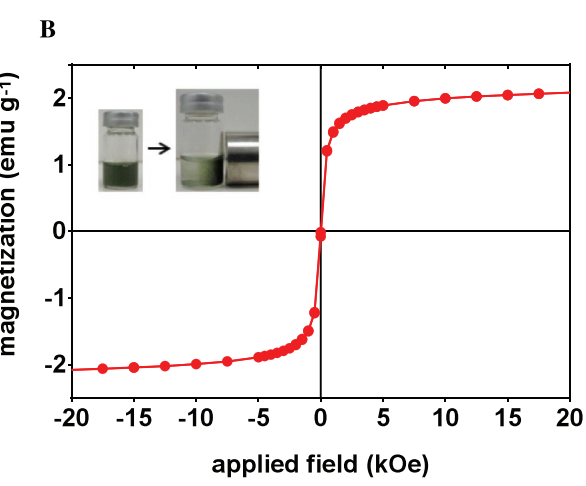

Figure 3. Magnetic responsiveness of MPs: A) TGA analysis of MPs and free-SPIONs for the quantification of SPIONs available in MPs. B) Magnetization curve as function of the applied magnetic field (Ms $=2.08$ emu $\mathrm{g}^{-1}$ ) confirming the superparamagnetic behavior of MPs. [Inset of (B)] Magnetic sorting of MPs from a water suspension using a permanent rare-earth magnet. 
Table 1. Results from anti-TB drug loading in the developed MPs.

\begin{tabular}{lccccc}
\hline$[\mathrm{P} 3]_{\text {initial }}\left[\mu \mathrm{g} \mathrm{mL} \mathrm{L}^{-1}\right]$ & $m_{\mathrm{P} 3 \text { initial }}[\mu \mathrm{g}]$ & Number of MPs & $m_{\mathrm{P} 3}$ in MPs ${ }^{\mathrm{a}}[\mu \mathrm{g}]$ & $m_{\mathrm{P} 3}$ per MPa) $[\mu \mathrm{\mu g} / \mathrm{MP}]$ & Loading efficiency $[\%]$ \\
\hline 21.4 & 37.5 & $(7.3 \pm 0.8) \times 10^{6}$ & $31.0 \pm 0.4$ & $(4.2 \pm 0.1) \times 10^{-6}$ & $82.7 \pm 0.9$ \\
\hline
\end{tabular}

a) Corresponds to the mean and two standard deviations of the mean of three independent experiments for a level of confidence of $95 \%$.

microparticulate drug carrier systems, e.g., fabricated by emulsion/solvent evaporation technique. ${ }^{[17]}$ The use of BSA as microgel filler was envisioned to improve the loading capacity of MPs for P3 by synergistically promoting a favorable drug/ matrix interaction while preventing the collapse of the MPs and further increase their total surface area. Moreover, the binding of hydrophobic molecules to BSA is reversible which allows the release of P3 from the MPs. ${ }^{[18]}$

\subsubsection{P3 Loaded MPs Physicochemical Characterization}

The specific surface area and the pore width of the P3 loaded MPs (P3-MPs), determined by the Brunauer-Emmett-Teller (BET) method, were $12.250 \mathrm{~m}^{2} \mathrm{~g}^{-1}$ (error of $\approx 2 \%$ ) and $2.183 \mathrm{~nm}$, respectively. Comparatively, the surface area of the P3-MPs is significantly higher than that of nonporous compact particles with an identical diameter $\left(0.8 \mathrm{~m}^{2} \mathrm{~g}^{-1}\right)^{[19]}$ and even higher than that of the original $\mathrm{CaCO}_{3}$ templates $\left(8.8 \pm 0.3 \mathrm{~m}^{2} \mathrm{~g}^{-1}\right) \cdot{ }^{[20]}$ Interestingly, P3-MPs also present a considerably higher specific surface area than other anti-TB drug loaded microparticles used for pulmonary drug delivery. For example, Sethuraman and Hickey ${ }^{[21]}$ reported that rifampicin-loaded PLGA microparticles had a specific surface area of $3.13 \mathrm{~m}^{2} \mathrm{~g}^{-1}$ while Tewes et al. ${ }^{[13 \mathrm{f}]}$ reported on inhalable trojan particles having a specific surface area in the range of $1.4-6.8 \mathrm{~m}^{2} \mathrm{~g}^{-1}$. The higher specific surface area of the developed P3-MPs is certainly related with the porous morphology of the template used to fabricate them and is reflected on its high drug loading efficiency and may result in improved aerodynamic properties. Collectively, these set of properties suggest that the developed system might have enhanced characteristics to be applied as anti-TB drug carriers aimed at pulmonary delivery.

The aerodynamic properties of the P3-MPs are critical for their deposition in the lower lung airways. In order to reach the alveoli the particles must have an aerodynamic diameter $\left(d_{\mathrm{a}}\right)$ in the range of $1-5 \mu \mathrm{m} .{ }^{[7]}$ The P3-MPs true density was determined by gas pycnometry and the value obtained was $1.49 \pm 0.02 \mathrm{~g} \mathrm{~cm}^{-3}$. The aerodynamic diameter $\left(d_{\mathrm{a}}\right)$ relates to the geometric diameter $\left(d_{\mathrm{g}}\right)$ as defined by the following equation ${ }^{[22]}$

$d_{\mathrm{a}}=d_{\mathrm{g}} \sqrt{\left(\frac{\rho_{\mathrm{p}}}{\rho_{0} \cdot \chi}\right)}$

where $\rho_{\mathrm{p}}$ is the apparent particle density, $\rho_{0}$ is the standard particle density $\left(1 \mathrm{~g} \mathrm{~cm}^{-3}\right)$, and $\chi$ is the particle shape factor that is 1 for spherical particles. It should be noted that the $\rho_{\mathrm{p}}$ can be lower than the material true density if the particle is porous as $\rho_{\mathrm{p}}$ is equal to the mass of a particle divided by its apparent volume that is the total volume of the particle excluding open pores but including closed pores. ${ }^{[22 \mathrm{~b}, 23]}$ Using the geometric diameter provided by SEM analysis $(2.86 \pm 0.04 \mu \mathrm{m})$ and applying the formula above, we obtained an aerodynamic diameter of $3.5 \pm 0.1 \mu \mathrm{m}$. However, the apparent density of the particles should be lower than the P3-MPs true density and consequently, the aerodynamic diameter of the P3-MPs should be smaller than the above estimated value, yet within the 1-5 $\mu \mathrm{m}$ range required to favor P3-MPs deposition in the lower airways. As a comparison with other inhalable dry powders incorporating SPIONs, dense microparticles prepared by spray-drying and composed of lactose, doxorubicin and about $10 \% \mathrm{w} / \mathrm{w}$ of SPIONs with a diameter of $1.6 \mu \mathrm{m}$ have an aerodynamic diameter of $3.3 \pm 1.7 \mu \mathrm{m}^{[15 \mathrm{a}]}$ and other based on mannitol with $13 \% \mathrm{w} / \mathrm{w}$ SPIONs with a diameter of $0.7 \mu \mathrm{m}$ have an aerodynamic diameter of $4.5 \pm 0.1 \mu \mathrm{m} .{ }^{[15 b]}$

\subsubsection{P3 Release Behavior}

The release profile of P3 from MPs was studied under different conditions simulating the physiological environments to which they can be exposed and to evaluate the functionality of their inherent magnetic responsive character. The release profile of P3 from the MPs into PBS at $37{ }^{\circ} \mathrm{C}$ is shown in Figure 4A. The P3 release occurs as a burst release in the first $6 \mathrm{~h}$ followed by a prolonged sustained release stage. During the first $6 \mathrm{~h}$, the burst release of P3 is higher in PBS 5.4 reaching 40\%, while in PBS 7.4 the release is more sustained reaching only $30 \%$. Independently of the solution, the initial burst may be mainly related with the release of the easily accessible P3 from MPs surface which is dominated by the solubility of the drug in the solvent and by convection processes (in this case, shaking which was kept constant through experiments).

The sustained release that follows is attributed to P3 diffusion through the polymeric matrix, ${ }^{[24]}$ clearly demonstrating the favorable interfacial interaction between the drug and the carrier (BSA and gelatin). P3 shows a faster release at acidic $\mathrm{pH}$ which can be explained by the higher solubility of P3 in acidic medium due to the protonation of nitrogen atoms in their purine-based structure (positive charge), as well as the lower electrostatic interactions between the positively charged P3 and the protein matrix due to the higher protonation of their carboxylic groups at acidic $\mathrm{pH}$. A similar $\mathrm{pH}$ triggered release behavior of positively charged drugs from microparticles and microcapsules was previously observed on other similar systems based on gelatin and BSA matrices. ${ }^{[10 c, e, 25]}$ Moreover, MPs morphology remains relatively intact during the release assay (Figure S1, Supporting Information), and therefore the matrix degradation/erosion phenomena are not expected to significantly affect P3 release kinetics. Note however that these assays were performed in PBS without the presence of proteolytic enzymes which will certainly accelerate the degradation rate of the gelatin/BSA matrix, and 


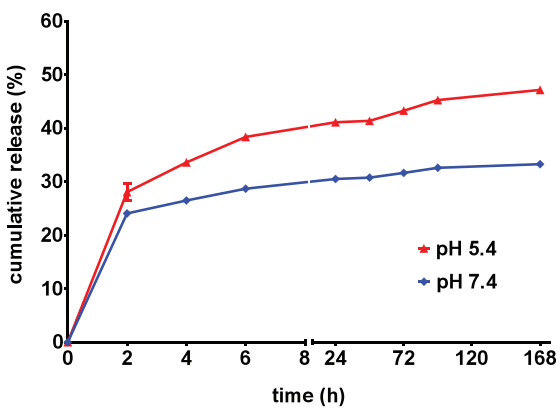

(A)

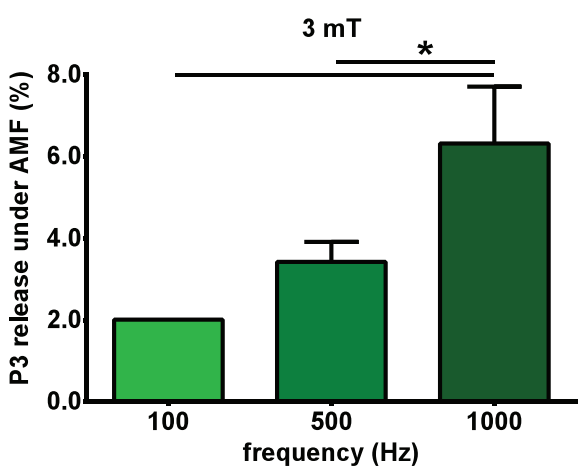

(C1)

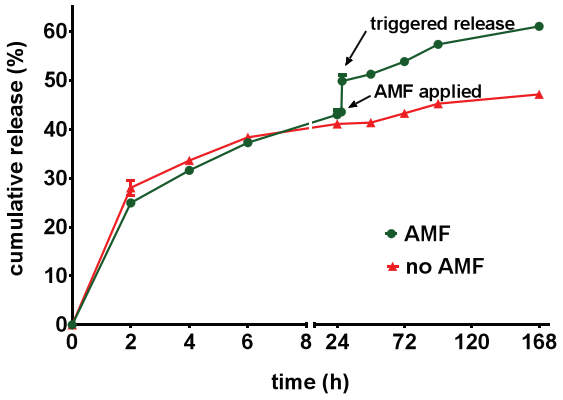

(B)

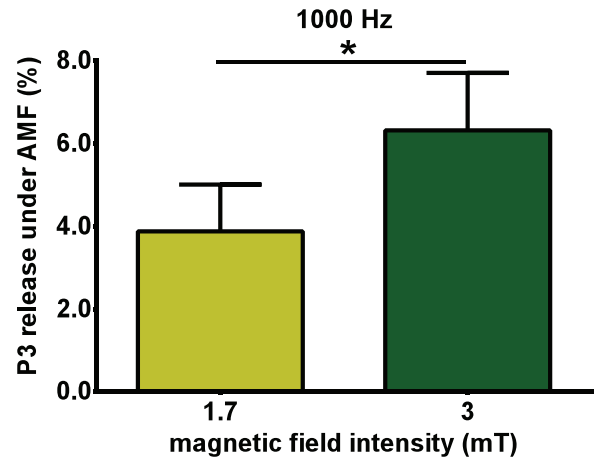

(C2)

Figure 4. A) Cumulative $\mathrm{P} 3$ release profile from the MPs in PBS at different $\mathrm{pH}$ [7.4 (blue), 5.4 (red)] and at $37^{\circ} \mathrm{C}$, for up to 7 days; values are represented by the mean with two standard deviations. B) Cumulative P3 release profile from the MPs in PBS 5.4 for 7 days under nonstimulation (red) and upon the actuation of an AMF of $1000 \mathrm{~Hz}, 3 \mathrm{mT}$ (green), for $30 \mathrm{~min}$ in the sustained release phase. Values are represented by mean with two standard deviations (level of confidence 95\%). C) Increment of P3 release due to application of AMF with different frequencies $(\mathrm{C} 1)$ and intensities (C2) in the sustained release phase and for $30 \mathrm{~min}$. Horizontal lines and * symbol denote statistically significant differences $(p<0.0001)$.

therefore, MPs erosion will have a relevant contribution for the P3 release kinetics in vivo. Since the $\mathrm{pH}$ of phagosomes varies from 4.5 to $6.2,,^{[16]}$ these results suggest that, it is likely that higher amounts of P3 drug are more prone to be released within these vesicles in infected macrophages, where $\mathrm{Mtb}$ resides and where $\mathrm{P} 3$ drug can be more effective.

In magnetic controlled drug delivery strategies, remote actuation on magnetic nanoparticles aims to generate heat by applying high frequency AMF producing a temperature-responsive change in the release profile of the system or mechanical deformations of the carrier matrix induced by a low frequency AMF that can modulate the release behavior and where the heat effect is negligible or nonexistent. ${ }^{[14 \mathrm{~d}]}$ In this work we explored this last approach. The effect of an AMF on the P3 release was studied by exposing P3-MPs for $30 \mathrm{~min}$ to a magnetic field with different intensities (1.7 and $3 \mathrm{mT}$ ) and frequencies (1000, 500, and $100 \mathrm{~Hz}$ ).

Considering the results obtained for P3 release in acidic $\mathrm{pH}$ and their relevance in the phagosome, the P3 release under AMF was assessed in PBS 5.4 and during the P3 sustained release phase. The sustained release phase was determined to be from $24 \mathrm{~h}$ to 7 days in accordance to the results shown in Figure 4A. Although no variation in solvent temperature was detected, the P3 release pattern was altered upon exposure to the AMF (Figure 4B), indicating a magnetically driven mechanical effect. An increase of $6.3 \% \pm 1.3 \%$ in the
P3 release was quantified after exposing the MPs to an AMF of $3 \mathrm{mT}$ and $1000 \mathrm{~Hz}$, while in the absence of AMF, the cumulative release was only $0.6 \% \pm 0.9 \%$. These results evidence that the applied AMF induces a tenfold increase in the P3 release. The percentage of P3 release due to the application of an AMF with different intensities and frequencies is represented in Figure 4C1,C2 where the Y-axis represents the increment observed in the cumulative release from the time point immediately before magnetic actuation and after this treatment. As can be seen from the shown data, there is an increase in the P3 release percentage with the increase in frequency and also with the increase of the magnetic field intensity. These results suggest that upon actuation of an AMF and in the presence of SPIONs, the P3-MPs offer the capability to trigger and modulate P3 release profile, which could be adjusted to meet the needs and duration of an anti-TB therapy. Furthermore, our results are in agreement with other particulate systems using MNPs for controlled drug release upon exposure to AMF. ${ }^{[14 a, 26]}$ For example, Guo et al. ${ }^{[26]}$ developed carboxymethyl dextrancoated magnetoliposomes with $6 \%$ of SPIONs and found that the release of doxorubicin could be modulated by exposure to low frequency AMF $(50 \mathrm{~Hz}, 15-45 \mathrm{mT})$. This study reported an increase in the release of doxorubicin with increasing intensity of the AMF without heat generation, suggesting that the triggered release under AMF was primarily due to the drug leakage resulting from the physical deformation of the liposomes. 

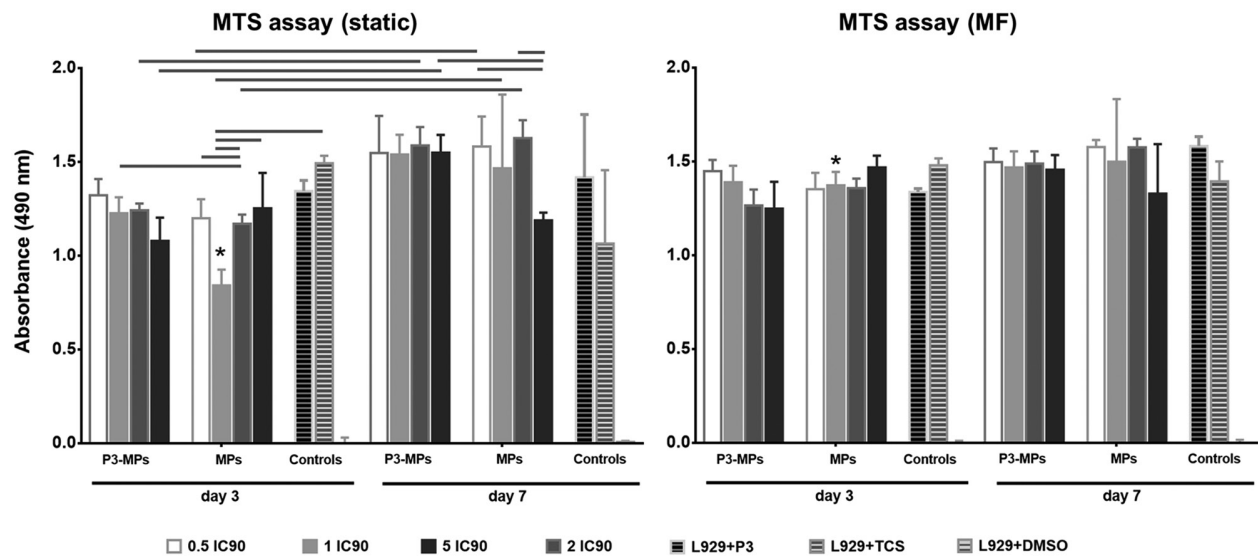

Figure 5. L929 metabolic activity cultured in the presence of P3-MPs and in MPs for 3 and 7 days in static and magnetic stimulation (MF) conditions assessed by MTS assay. Different inhibitory concentrations of P3 (0.5-5 IC90) were considered. Horizontal lines and * symbol denote statistically significant differences $(p<0.05)$. Moreover, L929+P3 represent P3 drug in MPs-free experimental control while L929+TCS (tissue culture coverslip) and L929+DMSO (dimethyl sulfoxide) represent the positive and negative control of cell viability, respectively.

\subsection{In Vitro Biological Assessment}

A cytotoxicity screening was performed in both MPs and P3-MPs using L929 cells for 3 and 7 days in static and MF cultures (Figure 5).

Overall, L929 in the presence of MPs and P3-MPs show viability values similar to cells cultured in MP-free conditions (L929+TCS). Moreover, there is an increase in L929 viability levels from day 3 to day 7 in static conditions, especially in conditions with increased number of MPs and in increased P3-MPs concentrations (2IC90 and 5IC90). As expected, culturing L929 cells under the influence of an external magnetic stimulation (2 Hz, $0.2 \mathrm{~mm}$ horizontal displacement) showed no significant differences $(p<0.05)$ in the metabolic activity of these cells in both MPs and P3-MPs groups, suggesting no detrimental effects of MF to L929 cells.

The MTS assay performed in THP-1 macrophages for up to 3 days in culture indicates that THP-1 metabolic activity is not affected by MPs, loaded or unloaded with P3 drug $(p<0.05)$ (Figure 6). Moreover, the concentration of MPs up to $5.9 \times 10^{5}$, which corresponds to 5IC90 in P3-MPs does not influence cell viability. This result confirms the noncytotoxic effect of MPs and P3-MPs to macrophage cells. The viability assay was also assessed in macrophages isolated from murine bone marrow. Overall, the absorbance values are lower than the ones detected for THP-1 cells and there is a decrease in cell viability from day 1 to day 3 in the presence of different concentrations of unloaded MPs $(p<0.05)$. Interestingly, when MPs are loaded with P3 drug, the values are maintained along the time in culture (Figure 6) and are increased in comparison to cell-MPs systems. These results suggest that although P3 drug was shown to have high anti-TB activity, ${ }^{[9 b]}$ it has no cytotoxic effect on murine bone marrow derived macrophages (mBMDM) when combined with the MPs. As for THP-1 cells, the MPs concentration and thus the amount of particles in contact with mBMDM, does not seem to influence cell viability levels. Moreover, after 3 days in culture, the metabolic activity levels of mBMDM cultured with P3-MPs are quite similar $(p<0.05)$ to MBMDM cultured in P3-MPs and MPs free conditions.

In order to determine the intracellular fate, MPs at different concentrations $\left(0.5-5 \times 10^{6}\right)$ were cultured with $\mathrm{mBMDM}$ in static conditions (Figure 7A). After $6 \mathrm{~h}$ incubation MPs are internalized by macrophages without impacting cell behavior, even at the highest concentration used. Moreover, confocal microscopy analysis confirmed the cellular uptake and localization of the MPs within macrophage cytoplasm as cell
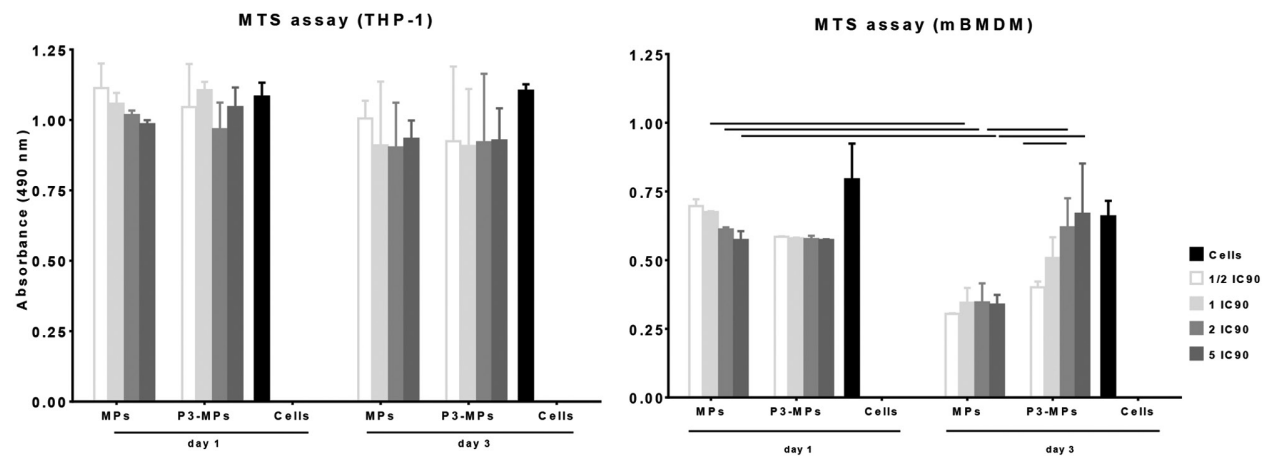

Figure 6. Metabolic activity of THP-1 and murine bone marrow derived macrophages (mBMDM) cultured in the presence of MPs and P3-MPs for 1 and 3 days. Different inhibitory concentrations of P3 (0.5-5 IC90) were considered. Horizontal lines denote statistically significant differences $(p<0.05)$. Cells represent macrophages cultured in the absence of P3-MPs or MPs. No significant differences were observed among conditions studied $(p<0.05)$. 

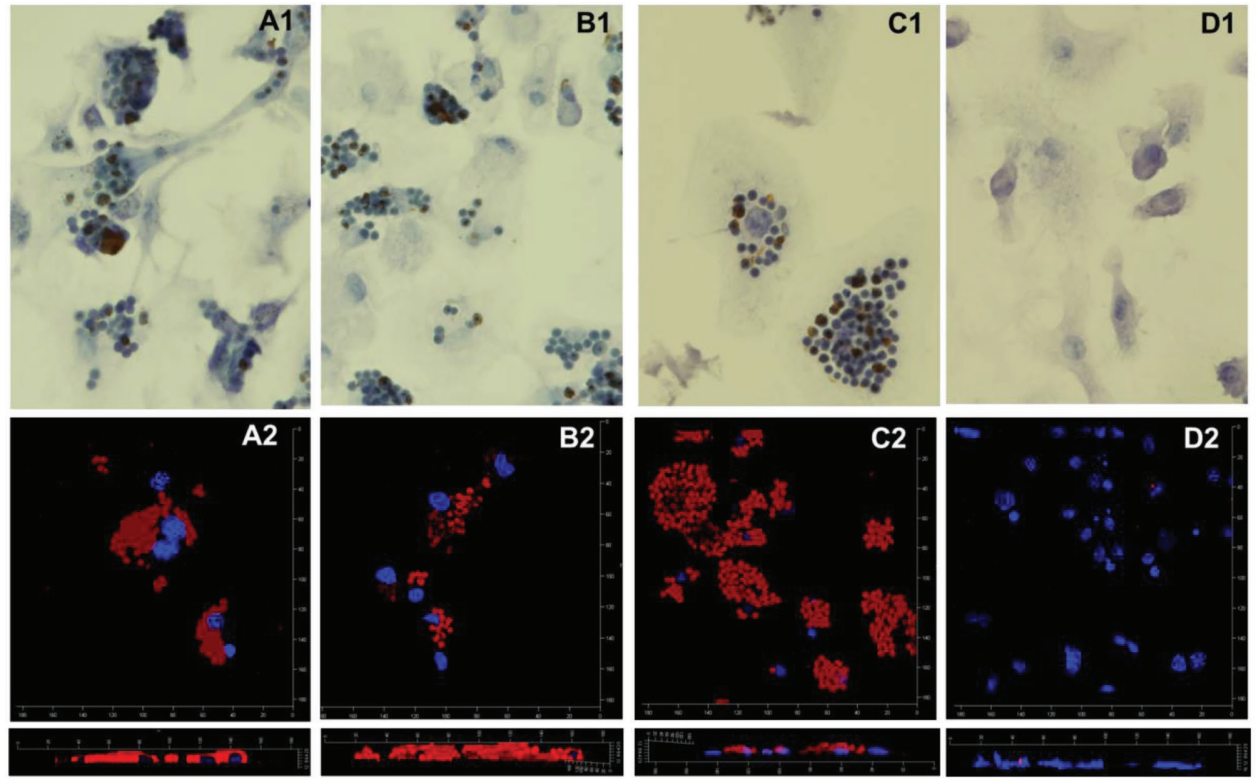

Figure 7. Assessment of MPs internalization by murine bone marrow derived macrophages (mBMDM). mBMDMs were cultured in different concentrations of MPs for $6 \mathrm{~h}$ and stained with HE (A1-D1, 40× magnification) or visualized in a confocal microscope (A2-D2). The letters, A), B), C) represent $0.5 \times 10^{6}, 2.5 \times 10^{6}$, and $5 \times 10^{6} \mathrm{MPs} \mathrm{mL}^{-1}$, respectively. Letter D) represents an MPs-free control. (A2-D2): blue represent cell nuclei stained with DAPI and red corresponds to the autofluorescence of the developed MPs. The bottom images are cross-sections of the 3D reconstructions of the focal planes of mBMDM cultured with MPs (scale bar represents $\mu \mathrm{m}$ ).

nuclei and MPs align in the same plan, confirming MPs internalization (Figure 7B). These results are in agreement with several publications that state macrophages phagocytosis exhibits an internalization peak around 2 to $3 \mathrm{~h}$ upon contact with a foreign body. ${ }^{[27]}$

In summary, our results suggest the potential of the designed MPs for magnetically actuated anti-TB therapies. MPs could be easily up scaled with the desired drug by adapting the cast method to a microfluidic platform in order to automatize and enable mass production of MPs. ${ }^{[28]}$ Likewise the applicability of MPs to the clinical area would include magnetotherapy devices that are relatively cheap and portable for generating the AMF and consequent activation of SPIONs to release the drug. Since the treatment requires medical monitoring, these devices could be available in healthcare facilities to which the patients would access periodically.

\section{Conclusions}

In this work we successfully designed a multifunctional microparticulate system with magnetic responsiveness for pulmonary delivery of an anti-TB drug candidate (P3). The P3-MPs were developed using a cast method and their physical and aerodynamic properties favor alveoli deposition and phagocytosis by alveolar macrophages. Additionally, the P3-MPs present a $\mathrm{pH}$ sensitive drug release profile, showing increased but sustained release rates in the acidic $\mathrm{pH}$ conditions of phagosomes, although P3 can also be released into the interstitial space in alveoli. Moreover, when an AMF was applied there was a tenfold increase in P3 release suggesting that an external AMF can remotely trigger and modulate drug release profile. These results may represent a major achievement for TB treatment as it would be possible to release a predetermined amount of drug at a predefined time and as often as needed according to the treatment requirements, thus improving treatment efficiency and patient compliance.

Overall, the developed MPs have shown potential to be used as inhalable drug carrier systems aiming at magnetically actuated anti-TB therapies and with particular added value as carriers for delivering hydrophobic anti-TB drugs, highlighting their versatile and functional potential for clinically relevant applications in pulmonary drug delivery.

\section{Experimental Section}

Materials: For MPs production and characterization the following materials were used: sodium carbonate $\left(\mathrm{Na}_{2} \mathrm{CO}_{3}, \geq 99.5 \%\right)$, gelatin from porcine skin (powder, gel strength $\approx 300 \mathrm{~g}$ Bloom, Type A), BSA (99\%), albumin-fluorescein isothiocyanate conjugate (BSA-FITC), ethylenediaminetetraacetic acid anhydrous (EDTA, 99.6\%), dimethyl sulfoxide (DMSO, $99.99 \%$ ), and PBS tablets and were purchased from Sigma-Aldrich; SPIONs in a aqueous dispersion (fluidMAG-UC/C:cationic charged, size $50 \mathrm{~nm}, \mathrm{w} / \mathrm{v}=25 \mathrm{mg} \mathrm{mL}$, polydispersity index 0.15 ) were purchased from Chemicell; genipin (99.7\%) was purchased from Commercial Rafer, S.L. and calcium chloride $\left(\mathrm{CaCl}_{2}, 100.2 \%\right)$ was purchased from VWR; glutaraldehyde was purchased from electron microscopy sciences and paraformaldehyde from Merck; $\mathrm{HCl}$ and $\mathrm{NaOH}$ $(1$ and $5 \mathrm{~m}$ ) were used for $\mathrm{pH}$ adjustments and were purchased from Fischer Chemical; ultrapure water was used in all experiments. For cell culture studies the following materials were used: DMEM (D2902, low glucose - with $1000 \mathrm{mg} \mathrm{L}^{-1}$ glucose and L-glutamine, without sodium bicarbonate and phenol red), DMEM (D5523, low glucose - with $1000 \mathrm{mg} \mathrm{L}^{-1}$ glucose and L-glutamine, without sodium bicarbonate, powder), sodium bicarbonate (100.0\%) and phorbol 12-myristate 13-acetate (PMA) were purchased from Sigma-Aldrich; fetal bovine 


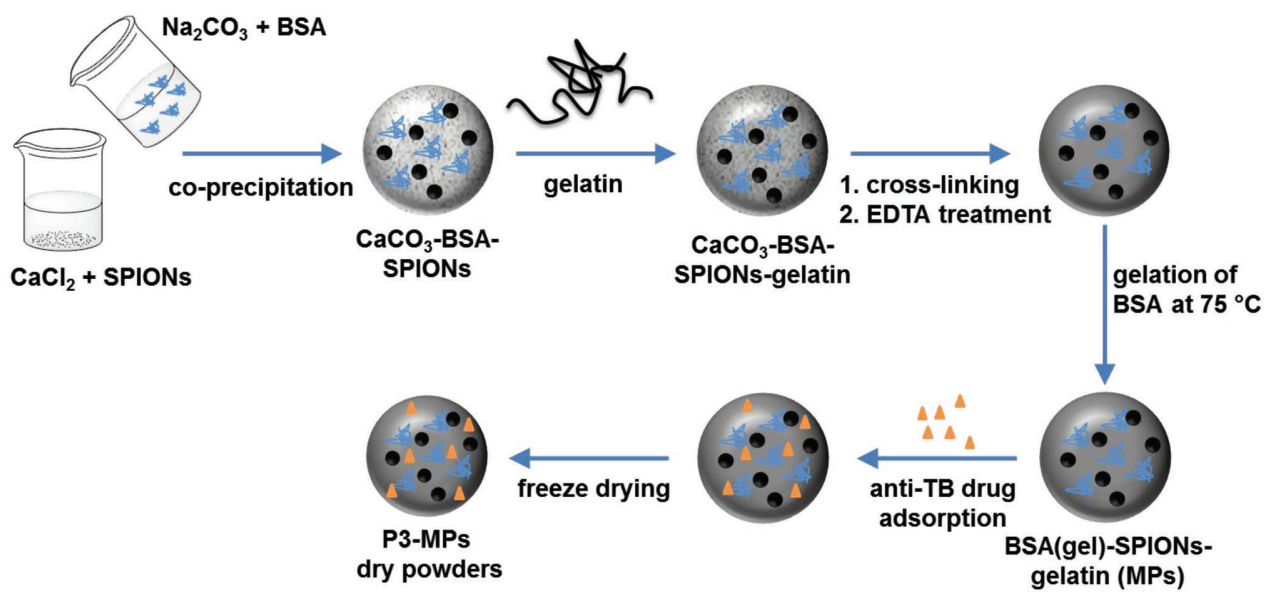

Figure 8. Production process of MPs and loading of anti-TB drug P3 within the produced MPs.

serum (FBS), HEPES, sodium pyruvate, L-glutamine, and PBS were purchased from Gibco, antibiotic/antimicotic solution from Alfagene and 4,6-diamidino-2-phenyindole dilactate (DAPI) from VWR.

Preparation of P3-MPs: The production process of P3-MPs is represented in Figure $8 . \mathrm{CaCO}_{3} \mathrm{MPs}$ incorporating SPIONs $\left(\mathrm{Fe}_{3} \mathrm{O}_{4}\right)$ and BSA were prepared by a coprecipitation reaction..$^{[10]}$ Commercial SPIONs $\left(500 \mu \mathrm{g} \mathrm{mL}^{-1}\right)$ were added to an aqueous solution of $\mathrm{CaCl}_{2}(0.33 \mathrm{M})$ and BSA $\left(500 \mu \mathrm{g} \mathrm{mL}^{-1}\right)$ was added to an aqueous solution of $\mathrm{Na}_{2} \mathrm{CO}_{3}$ $(0.33 \mathrm{M})$. The two mixtures were rapidly combined and the resultant mixture was heavily stirred (about $1000 \mathrm{rpm}$ ) for $30 \mathrm{~s}$ followed by a $15 \mathrm{~min}$ resting period. The supernatant was removed by centrifugation and the particles were washed three times with ultrapure water. Then, particles were suspended in a gelatin solution $\left(100 \mathrm{mg} \mathrm{mL}^{-1}\right)$ at $45^{\circ} \mathrm{C}$ for $6 \mathrm{~h}$ under mild agitation. Afterward, cold water was rapidly added. The mixture was centrifuged and washed with ultrapure water at least five times before suspending the particles in an aqueous genipin solution $(0.028 \mathrm{~m})$ overnight and under stirring. Then, particles were centrifuged and washed three times with ultra-pure water before being suspended in an EDTA solution $(0.2 \mathrm{M}, \mathrm{pH} \mathrm{5.5)}$ for $30 \mathrm{~min}$. The microgel particles were suspended in water and heated at $75{ }^{\circ} \mathrm{C}$ for 20 min under mild agitation before being incubated in an aqueous solution containing the anti-TB drug P3 $\left(25 \mu \mathrm{g} \mathrm{mL}^{-1}\right)$ for 2 days under agitation. For that, a P3 stock solution $\left(5 \mathrm{mg} \mathrm{mL}^{-1}\right)$ was first prepared with DMSO. A working solution of $25 \mu \mathrm{g} \mathrm{mL} \mathrm{g}^{-1}$ was prepared by diluting an appropriate volume of the P3 stock solution in water (final solution with $0.5 \% \mathrm{DMSO}$ ). The P3-MPs were then washed to remove unloaded drug and freeze dried to obtain dry powders. In the freeze-drying process, P3-MPs were flash frozen in liquid nitrogen to ensure that the MPs are frozen in suspension thus reducing the probability of aggregation and then dried under reduced pressure.

Characterization of Unloaded MPs: The dry powders obtained by freeze drying unloaded MPs were analyzed by SEM, field emission scanning electron microscope with focused ion beam (FIB-SEM), and by TEM.

For the SEM analysis, samples were placed on aluminum stubs with double-sided carbon tape and covered with gold under an argon atmosphere using a sputter coater (Cressington, 108A). The SEM (JSM-6010 LV, JEOL, Japan) was operated using an acceleration voltage of $15 \mathrm{kV}$. Elemental analysis was performed with an EDS (INCAx-Act, PentaFET Precision, Oxford Instruments).

The surface morphology of developed MPs was observed using a high-resolution FIB-SEM (Auriga Compact, Zeiss) at an acceleration voltage of $5.00 \mathrm{kV}$. For this analysis, the MPs were deposited onto glass supports $(\approx 1 \mathrm{~cm}$ diameter), mounted on aluminum stubs and coated with $1 \mathrm{~nm}$ of platinum under an argon atmosphere using a sputter coater (Leica EM ACE600).

TEM analysis was performed using a Jeol JEM 1400 equipment (EM 10C, Zeiss). Before analysis, dried MPs were fixed in a mixture of glutaraldehyde $(2.5 \% \mathrm{v} / \mathrm{v})$ and paraformaldehyde $(2 \% \mathrm{~m} / \mathrm{v})$ in $0.1 \mathrm{~m}$ cacodylate buffer. MPs were afterward washed with the same buffer and post-fixed with osmium tetroxide $(1 \% \mathrm{v} / \mathrm{v})$ in cacodylate buffer before being washed and dehydrated with increasing concentrations of ethanol. The MPs were then embedded with epoxy resin and thin sections were obtained by ultramicrotomy.

The shape and size of the MPs in ultrapure water (hydrated MPs) were analyzed by confocal laser scanning microscopy (Leica, model TCS SP8) using a laser excitation wavelength of $560 \mathrm{~nm}$ and taking advantage of MPs autofluorescence. The effect of $\mathrm{pH}$ on the diameter of the MPs has also been investigated by confocal microscopy with MPs dispersed in PBS at pH 7.4 (PBS 7.4) and at pH 5.4 (PBS 5.4).

Dried and hydrated MPs diameter measurements from SEM and confocal analysis were performed using software Image) (Image) $1.48 \mathrm{v}$ Wayne Rasband, NIH, USA, http://imagej.nih.gov/ij). The histogram of the frequency of diameter distribution and the corresponding normal distribution function has been derived from these assays for the dried and hydrated MPs.

To confirm the incorporation of BSA into the MPs, BSA-FITC, instead of BSA, was used to prepare MPs according to the procedure described in Preparation of P3-MPs. The BSA-FITC MPs were protected from light during the experimental setup and analyzed by confocal laser scanning microscopy with an excitation laser at $488 \mathrm{~nm}$ for the detection of FITC, a fluorescent chemical compound.

The size distribution of the MPs in aqueous solution was also qualitatively assessed by flow cytometry using the forward scatter detector for analyzing particle size and a side scatter detector for determining complexity of the MPs in an FACS ARIA cytometer (BD Biosciences).

The mass percentage of SPIONs incorporated into the unloaded MPs was determined by TGA with a simultaneous thermal analyzer (model STA7200, Hitachi). Dry MPs (2-4 mg) were placed in a platinum crucible, using an empty crucible as reference. The temperature of the furnace was raised to $105{ }^{\circ} \mathrm{C}$ at a rate of $10{ }^{\circ} \mathrm{C} \mathrm{min}-1$ and then isothermally held at this temperature for $10 \mathrm{~min}$, under a nitrogen atmosphere, to remove the strongly adsorbed water content. The temperature was then increased to $600{ }^{\circ} \mathrm{C}$ at $20^{\circ} \mathrm{C} \mathrm{min}-1$ under an oxygen atmosphere and isothermally held for $20 \mathrm{~min}$ in order to completely burn the organic component of MPs. The mass change events were recorded as function of the temperature. The mass percentage of SPIONs in the MPs was calculated from the difference between the initial mass of MPs and the total mass loss. Additionally, the water percentage of MPs corresponds to the mass loss of MPs until $105^{\circ} \mathrm{C}$

The magnetic properties of the MPs were assessed using an SQUID-VSM magnetometer (Quantum Design) in dry MPs with a mass of sample of $3.2 \mathrm{mg}$ at room temperature and under a magnetic field up to $20 \mathrm{kOe}$. For comparison purposes a study was also performed for the purchased SPIONs in the same conditions and using a mass of sample of $2.2 \mathrm{mg}$. 
Characterization of Developed P3-MPs: Assessment of P3 Loading Efficiency: About $7.3 \times 10^{6} \mathrm{MPs}$ were suspended in a P3 working solution prepared as described in Preparation of P3-MPs. The concentration of MPs $(2.9 \pm 0.8) \times 10^{7} \mathrm{MPs} \mathrm{mL}^{-1}$ was previously determined using disposable cell counting chambers (Hycor Biomedical Ltd) under a microscope (Leica DM750). The MPs were incubated in the P3 solution for 2 days at room temperature under mild agitation and protected from light. Afterward, MPs were collected by magnetic separation and the concentration of unloaded $\mathrm{P} 3$ in the supernatant was determined using a microplate reader (SYNERGY HT, Bio-tek instrum). The absorbance was measured at $420 \mathrm{~nm}$ and five readings were made for each assay. Three independent loading assays were performed. P3 concentration was determined using a calibration curve $\left(0,1,2.5,5,10,15,25 \mu \mathrm{g} \mathrm{m}^{-1}\right.$ of P3) and the loading efficiency was calculated as

\section{Loading efficiency $(\%)=(($ initial amount of $\mathrm{P} 3$-amount of $\mathrm{P} 3$ in supernatant $) /$ initial amount of $\mathrm{P} 3) \times 100$}

Physicochemical Characterization of P3-MPs: The BET specific surface area and the pore size distribution of the P3-MPs were determined with a Quantachrome Autosorb IQ2 instrument. The sample (mass of $68.5 \mathrm{mg}$ ) was outgassed at $50^{\circ} \mathrm{C}$ for $12 \mathrm{~h}$ before the measurements. The pore size distribution was calculated from the sorption isotherm using quenched solid state functional theory for slit-shaped pores.

The true density of P3-MPs was determined by gas pycnometry using an Ultrapyc 1200e gas pycnometer from Quantachrome Instruments. Analysis was performed at $25.2{ }^{\circ} \mathrm{C}$, with high purity nitrogen and $99.0 \mathrm{mg}$ of sample was used.

Assessment of Release Profiles: P3-MPs were suspended in $1 \mathrm{~mL}$ of either i) PBS 7.4 or ii) PBS 5.4 , at $37{ }^{\circ} \mathrm{C}$ and under mild shaking conditions. At defined time periods (2, 4, 6, 24, 48, 72, 96, $168 \mathrm{~h}), 800 \mu \mathrm{L}$ of the supernatant was collected, by magnetic separation of the particles, for assessing P3 concentration and an equal volume of fresh PBS with the specified $\mathrm{pH}$ was added. The absorbance of supernatant solution with released P3 was measured at $420 \mathrm{~nm}$ and PBS (7.4 or 5.4) was used as blank solution for background subtraction. Three independent release assays were performed for conditions i) and ii). The cumulative release of P3 was calculated using calibration curves obtained from standard solutions of P3 in PBS 7.4 and PBS 5.4 (minimum of six points were selected from 0 to $25 \mu \mathrm{g} \mathrm{mL} L^{-1}$ ).

Since MPs were envisioned as magnetic responsive carrier systems, the release profile of $\mathrm{P} 3$ was assessed under the actuation of an AMF. The AMF was generated in a custom-designed solenoid device ( 800 turns per meter, length: $31 \mathrm{~cm}$ and internal diameter: $4.8 \mathrm{~cm}$ ) by applying an alternating electric current with different intensities and frequencies.

First, the release assays of P3 from the MPs in PBS 5.4 medium was performed in the absence of an AMF (non-AMF) and P3 concentration was determined at defined time points: $2,4,6$, and $24 \mathrm{~h}$, using the procedure described above. The influence of AMF on P3 release was investigated selecting time points for magnetic actuation within the sustained release phase, previously assessed from the assays performed on the absence of AMF and determined to be from $24 \mathrm{~h}$ up to 7 days. In a typical experiment, the release assay proceeded under nonstimulated conditions up to $27 \mathrm{~h}$ and $\mathrm{P} 3$ release was assessed immediately before the application of the AMF. Then, the MPs were placed in the center of the solenoid and exposed to an AMF for $30 \mathrm{~min}$. Immediately after AMF stimulation, samples were taken from the solenoid and $\mathrm{P} 3$ release determined. Afterward the assay continued in the absence of AMF and P3 release was assessed at 48,72 , 96, and $168 \mathrm{~h}$. In order to study the influence of magnetic stimulation, different magnetic field intensities and frequencies were investigated. Magnetic fields of $1.7 \mathrm{mT}$ at $1000 \mathrm{~Hz}$ and $3 \mathrm{mT}$ at 100,500 , and $1000 \mathrm{~Hz}$ were investigated. Release profile assays were performed in triplicate for each frequency and magnetic field intensity analyzed.

Cytotoxicity Assessment of Developed P3-MPs: A cell line of mouse fibroblasts (L929) acquired from the European Collection of Cell Cultures was cultured in contact with different concentrations of P3-MPs for 1,3 , and 7 days. The cells were cultured in 96-well plates (Falcon) and grown as monolayers with a density of $1 \times 10^{5}$ cells cm $\mathrm{cm}^{-2}$ for $24 \mathrm{~h}$ in basal culture medium composed of DMEM with phenol red supplemented with $10 \% \mathrm{FBS}$ and $1 \%$ antibiotic/antimicotic solution. Then, culture medium was removed and replaced with culture medium with $6.2 \times 10^{3}$, $1.2 \times 10^{4}, 2.5 \times 10^{4}$, and $6.2 \times 10^{4} \mathrm{P} 3-\mathrm{MPs}$, corresponding to estimated P3 concentrations ranging from 0.15 to $1.5 \mu \mathrm{g} \mathrm{mL}^{-1}$. The selected range of $\mathrm{P} 3$ concentration corresponds to multiples of the inhibitory concentration IC90 ( $1 / 2$ IC90, 1 IC90, 2IC90, and 5IC90), which measures the effectiveness of $\mathrm{P} 3$ in inhibiting $90 \%$ of Mtb bacilli, as previously established. ${ }^{9 b]}$

Cell metabolic activity was assessed with MTS assay (Cell Titer 96 Aqueous Solution Cell Proliferation Assay, Promega). Briefly, cells were rinsed in PBS and the MTS solution was mixed with FBS-free and phenol red-free culture medium in a 1:5 ratio. Then, cells were incubated for $3 \mathrm{~h}$ at $37^{\circ} \mathrm{C}$ in a $5 \% \mathrm{CO}_{2}$ atmosphere. After this period, the supernatant was collected and the absorbance read at $490 \mathrm{~nm}$ (Synergy HT, Bio-TeK Instruments). The cell response to P3-MPs was assessed under a continuous magnetic field stimulation provided by a magnefect nanodevice (nanoTherics Ltd, UK) composed by an oscillating magnet array system $(2 \mathrm{~Hz}, 0.35 \mathrm{~T})$. Cells cultured under static conditions and in the presence of unloaded MPs or in contact with P3 drug without MPs were assessed as negative controls. Three independent assays with five replicates were considered.

Isolation and Differentiation of Macrophages: Isolation and Differentiation of Murine Bone Marrow Derived Macrophages (mBMDM): mBMDMs were isolated from 8 to 12-week-old C57BL/6 mice obtained from Charles River (Barcelona, Spain) housed under specific-pathogenfree conditions with food and water ad libitum at the Life and Health Sciences Research Institute (ICVS).

After mice euthanasia, the femurs and tibias were removed under aseptic conditions and flushed with DMEM supplemented with $10 \times 10^{-3} \mathrm{M}$ HEPES, $1 \times 10^{-3} \mathrm{M}$ sodium piruvate, $10 \times 10^{-3} \mathrm{M}$ L-glutamine and $10 \%$ heat-inactivated fetal bovine serum (complete DMEM). The resulting cell suspension was centrifuged and resuspended in complete DMEM with $20 \%$ of L929 cell conditioned medium (LCCM), at a final concentration of $5 \times 10^{5}$ cell mL $\mathrm{mL}^{-1}$. A volume of $8 \mathrm{~mL}$ was distributed per petri dish (Sterilin Limited), and cells were further incubated at $37{ }^{\circ} \mathrm{C}$ in a $5 \% \mathrm{CO}_{2}$ atmosphere. Four days after plating, $10 \mathrm{~mL}$ of complete DMEM supplemented with $20 \%$ of LCCM (a colonystimulating factor that promotes murine monocytes differentiation into macrophages) was added to the cells and at day 7 the cells were confirmed to be differentiated in macrophages. At this time, supernatants were discarded and cells were harvested gently with a cell scrapper in ice cold PBS. Thereafter, cells were centrifuged, distributed in tissue cultured 24-well plates at a density of $5 \times 10^{5}$ cell per well and incubated at $37^{\circ} \mathrm{C}$ in a $5 \% \mathrm{CO}_{2}$ atmosphere.

Culture and Differentiation of THP-1 Macrophages: Human macrophages derived from the cell line THP-1 were differentiated into macrophage-like cells by incubating the cells with phorbol 12-myristate 13-acetate (PMA, $50 \mathrm{ng} \mathrm{mL}^{-1}$ ) for 3 days. Afterward, cells were confirmed to be completely differentiated and were ressuspended in fresh media (DMEM) to a concentration of $2 \times 10^{5}$ cells per $\mathrm{mL}$. Then, THP-1 cells were plated on a tissue culture 24 well plate and incubated at $37^{\circ} \mathrm{C}$ in a $5 \% \mathrm{CO}_{2}$.

Viability of Macrophages ( $m B M D M$ and THP-1) in the Presence of MPs and P3-MPs: Both mBMDM and human THP-1 macrophages were incubated with different concentrations of $\mathrm{P} 3-\mathrm{MPs}$ corresponding to $1 / 2 \mid C 90,1 I C 90,2 I C 90$, and $5 I C 90$ dosage of $P 3$ drug. These P3 drug concentrations correlate in number to $5.9 \times 10^{4}, 1.2 \times 10^{5}, 2.3 \times 10^{5}$, $3.6 \times 10^{5}$, and $5.9 \times 10^{5}$ of unloaded MPs. Unloaded MPs were used as experimental controls. MPs and P3-MPs were tested in duplicates for each of the above multiples of IC90 concentrations and time point. The incubation times with MPs and P3-MPs were 24 and $72 \mathrm{~h}$ prior cell metabolic activity evaluation with MTS assay (Cell Titer 96 Aqueous Solution Cell Proliferation Assay, Promega) performed as described in Cytotoxicity Assessment of Developed P3-MPs.

Assessment of MPs Internalization by mBMDM: After the differentiation of mBMDM, as described in Isolation and Differentiation of Macrophages: Isolation and Differentiation of Murine Bone Marrow Derived Macrophages, $\mathrm{mBMDM}$ at a density of $5 \times 10^{5}$ cells per well were incubated in 24 well coverslips for $6 \mathrm{~h}$ with different amounts of MPs, corresponding to $0.5 \times 10^{6}, 2.5 \times 10^{6}$, and $5 \times 10^{6}$. 
After each time point, cells were rinsed with PBS, fixed and analyzed for HE staining using standard protocols or stained with 4,6-diamidino2-phenyindole dilactate (DAPI) for $10 \mathrm{~min}$. Samples were then observed and analyzed in an optical microscope (OLYMPUS BX61 microscope) and in a confocal laser scanning microscope (TCS SP8, Leica with a LASX software vs. 8), respectively.

Statistical Analysis: Data are represented by mean \pm two standard deviations for a level of confidence of $95 \%$ with the exception of in vitro cell culture assays whose data is represented by mean \pm one standard deviation.

Statistical analysis was carried out using a 2-Way ANOVA followed by the Tukey multiple comparison test to assess significant differences among studied conditions, using GraphPad Prism software (version 6). An exception was made for the release profile of P3 under an AMF. In this case statistical analysis was performed by one way ANOVA followed by Kruskal-Wallis multiple comparison test.

A minimum of $95 \%$ confidence interval was considered for all measurements $(p<0.05)$. The minimum number of samples and the number of independent assays are indicated in each quantitative assay performed. Statistical significant values and associated degree of confidence $(p<0.05)$ are denoted by the horizontal lines and symbols represented in the graphs.

\section{Supporting Information}

Supporting Information is available from the Wiley Online Library or from the author.

\section{Acknowledgements}

The authors wish to acknowledge the financial support from the Portuguese Foundation for Science and Technology (FCT) for the postdoctoral grant of M.S.M. (SFRH/BPD/110868/2015) and R.M.A.D (SFRH/BPD/112459/2015), FCT grant of E.T. (IF/01390/2014) and Recognize project (UTAP-ICDT/CTM-BIO/0023/2014). This article is also a result of the project "Accelerating tissue engineering and personalized medicine discoveries by the integration of key enabling nanotechnologies, marine-derived biomaterials and stem cells," supported by Norte Portugal Regional Operational Programme (NORTE 2020), under the PORTUGAL 2020 Partnership Agreement, through the European Regional Development Fund (ERDF). The authors acknowledge the financial support from the European Union Framework Programme for Research and Innovation HORIZON 2020, under the TEAMING Grant Agreement No. 739572 - The Discoveries CTR.

\section{Conflict of Interest}

The authors declare no conflict of interest.

\section{Keywords}

biomaterials, inhalable dry powders, magnetic drug targeting, microparticulate systems, remotely controlled drug release

Received: February 1, 2018 Published online: May 23, 2018

[1] W.H.O., http://www.who.int/features/factfiles/tuberculosis/en/ (accessed: January 2018).

[2] S. H. E. Kaufmann, Nat. Rev. Immunol. 2001, 1, 20.
[3] D.-D. Pham, E. Fattal, N. Tsapis, Int. J. Pharm. 2015, 478, 517.

[4] a) R. Sharma, D. Saxena, A. K. Dwivedi, A. Misra, Pharm. Res. 2001, 18, 1405; b) P. Muttil, J. Kaur, K. Kumar, A. B. Yadav, R. Sharma, A. Misra, Eur. J. Pharm. Sci. 2007, 32, 140.

[5] N. V. Satheesh Madhav, S. Kala, Int. J. PharmTech Res. 2011, 3, 1242.

[6] G. Pilcer, K. Amighi, Int. J. Pharm. 2010, 392, 1.

[7] E. Rytting, J. Nguyen, X. Wang, T. Kissel, Expert Opin. Drug Delivery 2008, 5, 629.

[8] a) A. Misra, A. J. Hickey, C. Rossi, G. Borchard, H. Terada, K. Makino, P. B. Fourie, P. Colombo, Tuberculosis 2011, 91, 71; b) K. Hirota, T. Hasegawa, H. Hinata, F. Ito, H. Inagawa, C. Kochi, G. Soma, K. Makino, H. Terada, J. Controlled Release 2007, 119, 69.

[9] a) C. Correia, M. A. Carvalho, M. F. Proença, Tetrahedron 2009, 65, 6903; b) A. C. R. Leite, Master Thesis, Minho University 2013.

[10] a) R. R. Costa, E. Castro, F. J. Arias, J. C. Rodríguez-Cabello, J. F. Mano, Biomacromolecules 2013, 14, 2403; b) R. R. Costa, C. A. Custódio, F. J. Arias, J. C. Rodríguez-Cabello, J. F. Mano, Nanomedicine 2013, 9, 895; c) A. Wang, Y. Cui, J. Li, J. C. M. van Hest, Adv. Funct. Mater. 2012, 22, 2673; d) X. Yan, J. Li, H. Möhwald, Adv. Mater. 2012, 24, 2663; e) H. Shen, H. Shi, M. Xie, K. Ma, B. Li, S. Shen, X. Wang, Y. Jin, J. Mater. Chem. B 2013, 1, 3906.

[11] a) S. Tiwari, A. P. Chaturvedi, Y. B. Tripathi, B. Mishra, AAPS PharmSciTech 2011, 12, 900; b) M. L. Manca, R. Cassano, D. Valenti, S. Trombino, T. Ferrarelli, N. Picci, A. M. Fadda, M. Manconi, J. Pharm. Pharmacol. 2013, 65, 1302.

[12] M. J. Hawkins, P. Soon-Shiong, N. Desai, Adv. Drug Delivery Rev. 2008, 60, 876

[13] a) P. Dames, B. Gleich, A. Flemmer, K. Hajek, N. Seidl, F. Wiekhorst, D. Eberbeck, I. Bittmann, C. Bergemann, T. Weyh, L. Trahms, J. Rosenecker, C. Rudolph, Nat. Nanotechnol. 2007, 2, 495; b) G. Hasenpusch, J. Geiger, K. Wagner, O. Mykhaylyk, F. Wiekhorst, L. Trahms, A. Heidsieck, B. Gleich, C. Bergemann, M. K. Aneja, C. Rudolph, Pharm. Res. 2012, 29, 1308; c) A. Krafcik, P. Babinec, I. Frollo, J. Magn. Magn. Mater. 2015, 380, 46; d) O. Pourmehran, M. Rahimi-Gorji, M. Gorji-Bandpy, T. B. Gorji, J. Magn. Magn. Mater. 2015, 393, 380; e) N. A. Stocke, S. A. Meenach, S. M. Arnold, H. M. Mansour, J. Z. Hilt, Int. J. Pharm. 2015, 479, 320; f) F. Tewes, C. Ehrhardt, A. M. Healy, Eur. J. Pharm. Biopharm. 2014, 86, 98.

[14] a) N. Griffete, J. Fresnais, A. Espinosa, C. Wilhelm, A. Bee, C. Menager, Nanoscale 2015, 7, 18891; b) S.-H. Hu, S.-Y. Chen, X. Gao, ACS Nano 2012, 6, 2558; c) M. Nair, R. Guduru, P. Liang, J. Hong, V. Sagar, S. Khizroev, Nat. Commun. 2013, 4, 1707; d) A. K. Hauser, R. J. Wydra, N. A. Stocke, K. W. Anderson, J. Z. Hilt, J Controlled Release 2015, 219, 76.

[15] a) A. A. McBride, D. N. Price, L. R. Lamoureux, A. A. Elmaoued, J. M. Vargas, N. L. Adolphi, P. Muttil, Mol. Pharmaceutics 2013, 10, 3574; b) N. A. Stocke, S. A. Meenach, S. M. Arnold, H. M. Mansour, J. Z. Hilt, Int. J. Pharm. 2015, 479, 320; c) M. Dierendonck, S. De Koker, C. Cuvelier, J. Grooten, C. Vervaet, J.-P. Remon, B. G. De Geest, Angew. Chem., Int. Ed. 2010, 49, 8620; d) R. Diab, J. Brillault, A. Bardy, A. V. Gontijo, J. C. Olivier, Int. J. Pharm. 2012, $436,833$.

[16] O. H. Vandal, C. F. Nathan, S. Ehrt, J. Bacteriol. 2009, 191, 4714.

[17] T. Parumasivam, S. S. Leung, D. H. Quan, J. A. Triccas, W. J. Britton, H. K. Chan, Eur. J. Pharm. Sci. 2016, 88, 1.

[18] a) K. Paál, J. Müller, L. Hegedûs, Eur. J. Biochem. 2001, 268, 2187; b) M. Purcell, J. F. Neault, H. A. Tajmir-Riahi, Biochim. Biophys. Acta, Protein Struct. Mol. Enzymol. 2000, 1478, 61.

[19] G. B. Sukhorukov, D. V. Volodkin, A. M. Gunther, A. I. Petrov, D. B. Shenoy, H. Mohwald, J. Mater. Chem. 2004, 14, 2073.

[20] D. V. Volodkin, A. I. Petrov, M. Prevot, G. B. Sukhorukov, Langmuir 2004, 20, 3398

[21] V. V. Sethuraman, A. J. Hickey, AAPS PharmSciTech 2002, 3, 7. 
[22] a) B. Y. Shekunov, P. Chattopadhyay, H. H. Y. Tong, A. H. L. Chow Pharm. Res. 2007, 24, 203; b) P. F. DeCarlo, J. G. Slowik, D. R. Worsnop, P. Davidovits, J. L. Jimenez, Aerosol Sci. Technol. 2004, 38, 1185.

[23] F. Tewes, C. Ehrhardt, A. M. Healy, Eur. J. Pharm. Biopharm. 2014, 86, 98.

[24] P. O'Hara, A. J. Hickey, Pharm. Res. 2000, 17, 955.

[25] H. J. Shen, H. Shi, K. Ma, M. Xie, L. L. Tang, S. Shen, B. Li, X. S. Wang, Y. Jin, Acta Biomater. 2013, 9, 6123.
[26] a) H. Guo, W. Chen, X. Sun, Y.-N. Liu, J. Li, J. Wang, Carbohydr. Polym. 2015, 118, 209; b) P. M. Peiris, L. Bauer, R. Toy, E. Tran, J. Pansky, E. Doolittle, E. Schmidt, E. Hayden, A. Mayer, R. A. Keri, M. A. Griswold, E. Karathanasis, ACS Nano 2012, 6, 4157.

[27] a) G. J. Cannon, J. A. Swanson, J. Cell Sci. 1992, 101, 907; b) A. Gupta, G. Pant, K. Mitra, J. Madan, M. K. Chourasia, A. Misra, Mol. Pharmaceutics 2014, 11, 1201.

[28] D. Dendukuri, P. S. Doyle, Adv. Mater. 2009, 21, 4071. 